\title{
Haemostatic Response of Polyethylene Terephthalate Treated by Oxygen and Nitrogen Plasma Afterglows
}

\author{
Metod Kolar ${ }^{1}$ and Gregor Primc ${ }^{2}$ \\ ${ }^{1}$ Centre of Excellence, PoliMat, Tehnološki Park 24, 1000 Ljubljana, Slovenia \\ ${ }^{2}$ Plasmadis d.o.o., Teslova 30, 1000 Ljubljana, Slovenia \\ Correspondence should be addressed to Gregor Primc; gregor.primc@plasmadis.com
}

Received 13 November 2015; Accepted 24 December 2015

Academic Editor: Yakai Feng

Copyright ( 2016 M. Kolar and G. Primc. This is an open access article distributed under the Creative Commons Attribution License, which permits unrestricted use, distribution, and reproduction in any medium, provided the original work is properly cited.

\begin{abstract}
Samples of polymer polyethylene terephthalate were coated with heparin and the haemostatic response has been determined by optical imaging of samples after incubation with fresh blood from a healthy donor. Prior to coating the samples were treated by neutral reactive particles of the oxygen or nitrogen plasma flowing afterglow. X-ray photoelectron spectroscopy analysis showed intensive functionalization of the polymer foils upon treatment with afterglows; however, the concentration of sulphur from heparin remained below the detection limit. The optical imaging showed densely distributed blood platelets in highly activated forms on untreated samples, whereas treatment with both afterglows revealed improved hemocompatibility. Best results were obtained for oxygen-functionalized polymer, whereas additional coating with heparin caused moderate loss of hemocompatibility, that was explained by deactivation of surface functional groups upon incubation with heparin.
\end{abstract}

\section{Introduction}

Polymer materials are widely used for manufacturing products that come to contact with body tissues and liquids $[1,2]$. The biological response depends enormously on the type of polymer as well as its surface finish. In many cases the biological response is not adequate so the products should be covered with thin coatings of materials of better biocompatibility $[3,4]$. Examples of particular interest are products that come into contact with human blood, such as artificial blood vessels, fistulae, vascular stents, and heart valves. Such products are made from a limited number of polymers such as polytetrafluoroethylene (PTFE), polyurethane (PU), and polyethylene terephthalate (PET) [5-7]. The type of polymer is chosen according to mechanical properties and chemical stability, whereas the biocompatibility should be reasonably good. As-synthesized polymers never exhibit excellent biocompatibility when in contact with human blood, so many commercial cardiovascular implants are covered with different coatings (i.e., heparin) in order to improve the hemocompatibility $[6,7]$. The coatings, however, do not always persist after prolonged incubation with human blood.
They either can interact chemically with blood constituents or are just dissolved and washed away. Although numerous attempts have been made to stabilize such coatings on polymer surfaces, the results are still not optimal $[6,8]$.

An alternative to coating of polymer materials that come into contact with human blood is nanostructuring and functionalization [3,9-14]. Such treatments are often performed in gaseous plasma [15]. Gaseous plasma definitely causes rich functionalization of polymer surfaces with desired functional groups [16]; however, on the other hand it also causes modification of subsurface properties of polymer materials. Namely, gaseous plasma is rich in charged particles and neutral reactive particles that may be excited to metastables of high potential energy. Such particles, when deexcited, radiate in the entire spectrum from IR to VUV [17]. Oxygen plasma, for example, is a source of VUV radiation that comes from radiative transition of neutral oxygen atoms. On the other hand, nitrogen plasma exhibits UV radiation from different transitions of both neutral and positively charged nitrogen molecules. Such radiation is absorbed in the subsurface film of polymer and causes bond scissions which in turn cause modification of chemical and mechanical 
properties of polymers treated by such plasma [18]. In order to avoid this effect but still enable functionalization with specific functional groups, it is often better to use flowing afterglow of appropriate gaseous plasma. Such an approach has been also adopted in our case and the results show excellent hemocompatibility at practically no modification of subsurface polymer film.

\section{Experimental Setup}

2.1. Plasma Treatment. Samples of semicrystalline polymer PET (Goodfellow Ltd.) were treated in the experimental system presented schematically in Figure 1. Samples were placed into the treatment chamber which was made from borosilicate glass. Neutral reactive particles were leaked into the treatment chamber through a rather narrow quartz tube of an inner diameter $6 \mathrm{~mm}$. The quartz tube was mounted into a microwave (MW) cavity where plasma was created. The cavity was powered with a MW generator of a standard frequency $2.45 \mathrm{GHz}$ and an adjustable power up to $300 \mathrm{~W}$. Due to continuous leakage of gas through the flow controller on one side of the quartz tube and pumping of the treatment chamber on the other side, there was a pressure gradient along the tube which allowed for fast drifting of gas from the plasma to the treatment chamber. The sample was placed in such a manner that any radiation from gaseous plasma could not reach the samples. The samples were treated in oxygen and nitrogen plasma. The concentration of neutral gaseous atoms in the treatment chamber was estimated with a catalytic probe, which was mounted onto the treatment chamber as shown schematically in Figure 1. Plasma was created at the MW power of $150 \mathrm{~W}$ and gas flow of $140 \mathrm{sccm}$. In such conditions the pressure in the treatment chamber was $50 \mathrm{~Pa}$. In case the working gas was oxygen, the concentration of neutral oxygen atoms in the ground state at the position next to the sample was approximately $7 \times 10^{20} \mathrm{~m}^{-3}$ [19], whereas, in the case of nitrogen, the dissociation fraction was somehow lower and the $\mathrm{N}$-atom density was only approximately $4 \times$ $10^{20} \mathrm{~m}^{-3}$. The treatment time for both cases was $30 \mathrm{~s}$.

2.2. XPS Surface Characterization. The samples were characterized with X-ray photoelectron spectroscopy (XPS) within a few minutes after the treatment. XPS characterization was performed using an XPS (TFA XPS Physical Electronics, Münich, Germany). The samples were excited with monochromatic $\mathrm{Al} \mathrm{K} \alpha_{1,2}$ radiation at $1486.6 \mathrm{eV}$ over an area with a diameter of $400 \mu \mathrm{m}$. Photoelectrons were detected with a hemispherical analyser positioned at an angle of $45^{\circ}$ with respect to the normal of the sample surface. XPS survey spectra were measured at pass energy of $187 \mathrm{eV}$ using an energy step of $0.4 \mathrm{eV}$, whereas high-resolution spectra were measured at pass energy of $23.5 \mathrm{eV}$ using an energy step of $0.1 \mathrm{eV}$. An additional electron gun was used for surface neutralization during XPS measurements. All spectra were referenced to the main C1s peak of the carbon atoms, which was assigned a value of $284.8 \mathrm{eV}$. The measured spectra were analysed using MultiPak v8.1c software (Ulvac-Phi, Inc.,

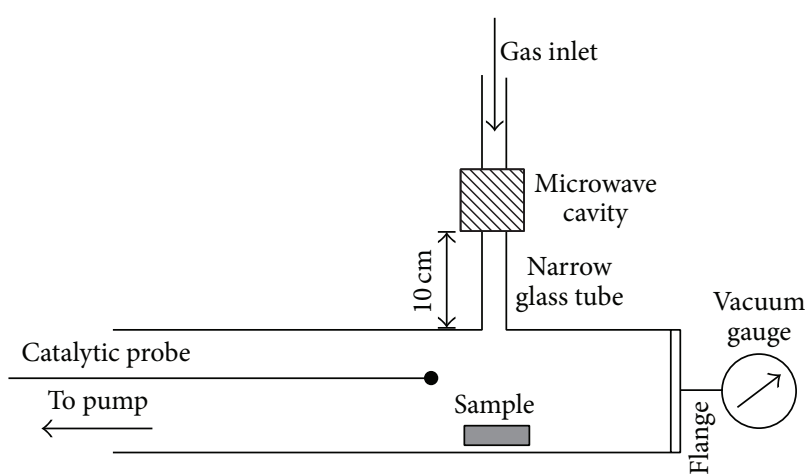

FIGURE 1: The experimental setup.

Kanagawa, Japan, 2006) from Physical Electronics, which was supplied with the spectrometer.

2.3. Coating with Heparin. Selected samples were incubated in a solution containing $1 \%$ heparin in $50 \mathrm{mM}$ MES buffer. In order to facilitate covalent binding of heparin to the polymer surface treated in nitrogen plasma, a crosslinking agent was added at the concentration of $6 \mathrm{mM}$. The agent was 1-ethyl3-(3-dimethylaminopropyl)carbodiimide hydroxide (EDC). EDC is used for coupling of amino groups on the nitrogen plasma-treated polymer surface with carboxyl groups of heparin $[20,21]$. The binding was stabilized using Nhydroxysulfosuccinimide (NHS) at the concentration of $3.5 \mathrm{mM}$. The samples were left in the solution for at least 4 hours. Then they were rinsed thoroughly, first with $3 \mathrm{M}$ water solution of $\mathrm{NaCl}$ and then by Milli-Q water. Samples were then dried in ambient conditions. For the polymer treated in oxygen plasma we used an anchoring agent polyethylenimine (PEI) which is rich in amino groups that are coupled to the oxygen plasma-treated surfaces by electrostatic interactions [21]. All reagents were purchased from Sigma Aldrich.

2.4. Platelet Adhesion and Characterization. Both plasmatreated and heparin coated samples were incubated with fresh blood from a healthy donor, which was supplied from the Blood Transfusion Centre of Slovenia. Blood was withdrawn with 21-gauge needle into evacuated tube containing 3.2\% sodium citrate as anticoagulant. Samples were incubated with $1 \mathrm{~mL}$ of fresh whole human blood in 24-well cell culture plate with shaking at $250 \mathrm{RPM}$ for $1 \mathrm{~h}$ at $37^{\circ} \mathrm{C}$. After incubation the samples were rinsed several times with phosphate buffer saline (1x PBS) in order to remove blood residues and treated with $2.5 \%$ glutaraldehyde. After fixation the samples were rinsed with deionized water and left to air-dry. All experiments were performed in triplicate. The adhesion of platelets on samples was observed by confocal microscopy. A high-resolution Axio CSM 700 (Carl Zeiss, Jena, Germany) confocal light microscope was used.

\section{Results and Discussion}

Treatment of polymer samples with neutral reactive particles created in plasma caused activation of the sample surface due 
TABLE 1: XPS surface composition of PET samples coated with heparin (in at.\%).

\begin{tabular}{llcccc}
\hline Number & Sample & $\mathrm{C}$ & $\mathrm{O}$ & $\mathrm{N}$ & $\mathrm{S}$ \\
\hline$\# 0$ & Pure untreated PET & 75.1 & 24.9 & $/$ & $/$ \\
$\# 1$ & Untreated + heparin & 73.1 & 26.9 & $/$ & $/$ \\
$\# 2$ & Treated in $\mathrm{O}_{2}$ plasma & 59.5 & 40.5 & $/$ & $/$ \\
$\# 3$ & Treated in $\mathrm{O}_{2}$ plasma + heparin & 62.5 & 35.6 & 1.9 & $/$ \\
$\# 4$ & Treated in $\mathrm{N}_{2}$ plasma & 66.2 & 30.2 & 3.6 & $/$ \\
$\# 5$ & Treated in $\mathrm{N}_{2}$ plasma + heparin & 61.3 & 35.5 & 3.2 & $/$ \\
\hline
\end{tabular}

to functionalization with specific functional groups. Figure 2 reveals XPS spectra of the samples treated by either oxygen or nitrogen atoms in the treatment chamber presented in Figure 1 and incubated with heparin. The peaks corresponding to different elements in the surface film of samples changed upon the treatment and a small peak corresponding to nitrogen is observable at the binding energy of $400 \mathrm{eV}$. The spectra presented in Figure 2 allow for determination of elemental composition in the surface film of thickness of several nanometres. The results are summarized in Table 1. The composition for uncoated samples is added for comparison. The concentration of oxygen on untreated PET incubated with heparin (sample \#1) is slightly larger than for pure PET (sample \#0) that is explained by a high concentration of oxygen in the polysaccharide. The difference, however, is not dramatic: instead of 25 at.\% typical for pure PET the concentration of oxygen for the untreated polymer incubated with heparin is 27 at.\%. Furthermore, the concentration of both nitrogen and sulphur which should be present in heparin is below the detection limit of XPS. Such results indicate that heparin did not adhere well to the surface of untreated PET and was rather washed away upon preparation procedure.

The concentration of oxygen on polymer treated with neutral oxygen atoms (sample \#2) is much larger than on pure untreated PET material (sample \#0). This result is expected because neutral oxygen atoms interact extensively with polymer materials placed into the treatment chamber for time as long as $30 \mathrm{~s}$ [16]. In fact, functionalization of aromatic polymers in oxygen plasma afterglow is even better than in glowing plasma itself [22]. Because the $\mathrm{O}$-atom density is approximately $7 \times 10^{20} \mathrm{~m}^{-3}$, the corresponding flux of $\mathrm{O}$ atoms is approximately $10^{23} \mathrm{~m}^{-2} \mathrm{~s}^{-1}$ and the fluence $10^{24} \mathrm{~m}^{-2}$. This is a huge value taking into account the surface density of atoms in the solid material which is of the order of $10^{19} \mathrm{~m}^{-2}$. More interesting is the concentration of oxygen on the surface of the sample first treated with oxygen atoms and then incubated with heparin (sample \#3). The concentration is approximately 35 at.\% that is somehow lower than for the oxygen-treated sample (\#2), but well above the untreated sample $(\# 0)$. Such a large concentration can be explained either by polymer functionalization with O-rich functional groups upon treatment with neutral oxygen atoms or by presence of heparin or both. Due to the fact that the concentration of nitrogen on this sample is measurably high, one can speculate that the excessive oxygen on this sample is

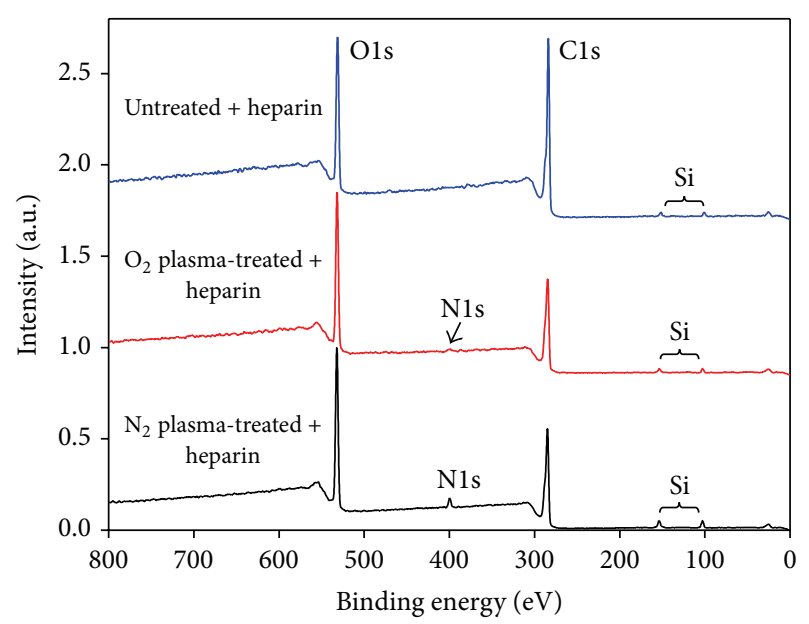

FIGURE 2: XPS survey spectra of PET samples coated with heparin.

at least partly due to the presence of heparin on the polymer, which was pretreated by neutral oxygen atoms.

Such a speculation is supported from the composition of the sample which was pretreated by nitrogen atoms and incubated with heparin (sample \#5). Namely, in this case the concentration of oxygen is nearly identical as on the sample treated by oxygen atoms and incubated with heparin (sample $\# 3$ ). Because the pretreatment of this sample was performed in nitrogen atmosphere, it is clearly not possible that the increased concentration of oxygen is a result of pretreatment, but it is rather due to the presence of heparin on such a surface.

Modifications of surface composition between polymer materials with different pretreatment are reflected in the ability for blood platelets to adhere to and activate on the polymer surface. Figures 3-7 show both low and high magnification optical images of the samples after incubation with human blood. One can observe huge differences which will be discussed below. An untreated sample incubated with heparin (Figure 3 ) is nearly entirely covered with blood platelets. The high magnification image reveals some details about the morphological shape. Numerous flakes observed in the image are blood platelets in highly activated (often called "fully spread") form [23]. The sample which was not plasma pretreated is therefore as thrombogenic as any other polymer material, although it has been incubated with heparin. This result is sound with the result obtained by XPS. Namely, XPS showed only a marginal increase of oxygen on heparin-incubated sample and no nitrogen, so it was possible to speculate that any heparin was washed away even before incubation with human blood. The polymers without any antithrombogenic surface finish therefore adhere blood platelets and cause their activation as observed in Figure 3.

Figure 4 represents optical images of the sample treated by oxygen atoms only and not incubated with heparin. The treatment obviously causes a dramatic improvement of the polymer hemocompatibility. Low magnification image reveals sparsely distributed dots which are actually individual blood platelets that becomes apparent after viewing the high-resolution image. The treatment of PET material by 


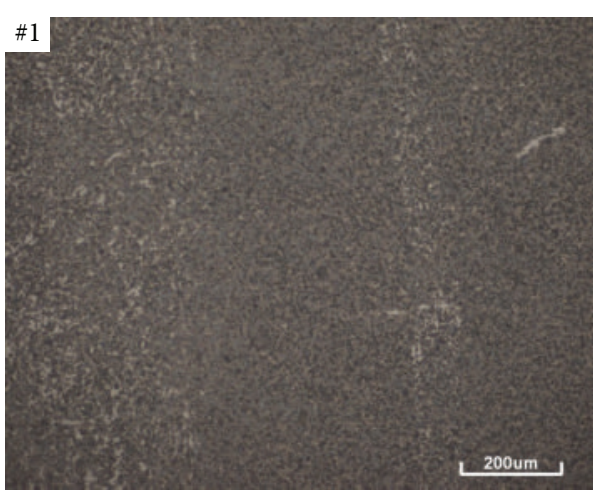

(a)

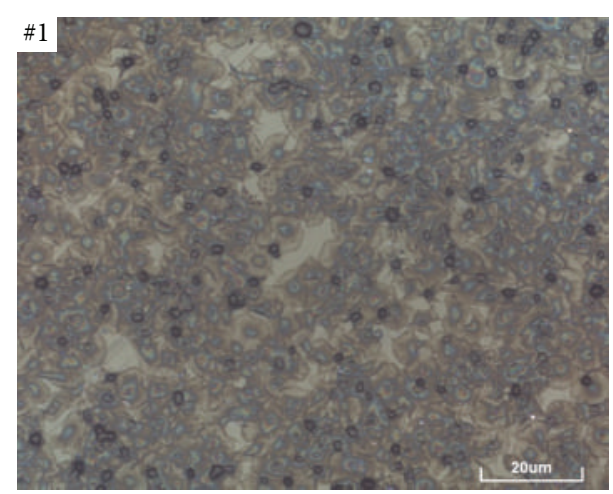

(b)

FIGURE 3: Optical image of platelets on surface of untreated polymer PET (\#1) coated with heparin after incubation with fresh blood at two different magnifications ((a) and (b)).

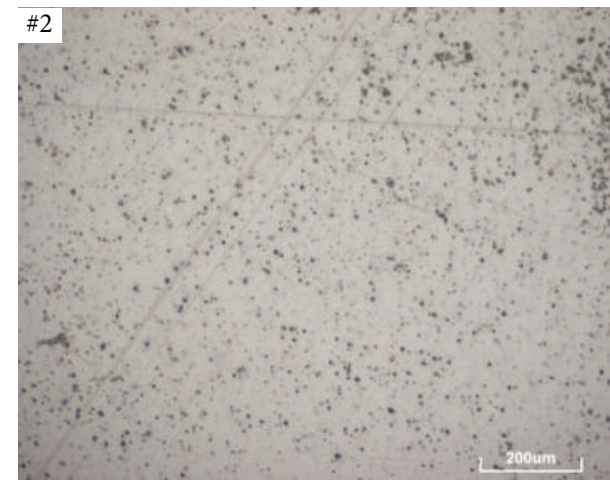

(a)

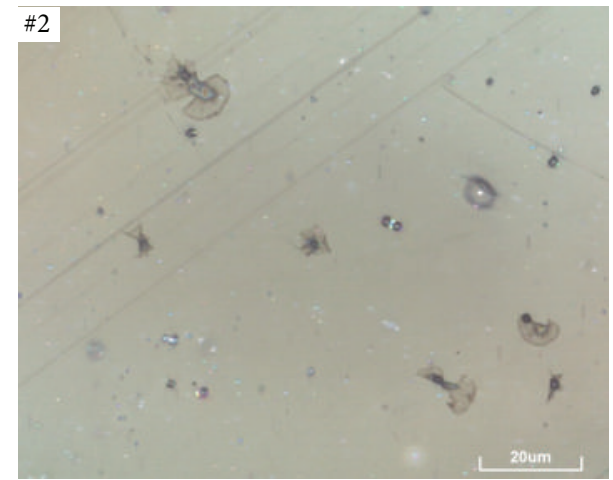

(b)

FIGURE 4: Optical image of platelets on surface of PET polymer treated in oxygen plasma (\#2) after incubation with fresh blood at two different magnifications ((a) and (b)).

neutral oxygen atoms is therefore beneficial and results are similar to those obtained by treatment of this polymer in oxygen plasma $[24,25]$. The poor ability for adhesion of blood platelets on oxygen-functionalized materials has been explained by different conformation of blood proteins that adhere to the surface of PET polymer in a fraction of a second after incubating with fresh blood, so well before any platelet adheres and activates [26].

Incubation of the sample treated by oxygen atoms with heparin does not modify the hemocompatibility of this sample much. This is obvious from Figure 5 which is very similar to Figure 4. The only difference is in the high-resolution images. Whereas in Figure 4(a) one can observe only separate platelets, Figure 5(a) reveals clusters. Clusters contain both highly and weakly activated platelets. As mentioned above the activation degree is revealed from the morphology of the platelet. Almost spherical platelets are not activated, whereas flakes of rather large diameter indicate platelets in the final state of activation. In between there are flakes in dendritic or spread dendritic forms. The incubation of oxygen-treated polymer with heparin, therefore, does not improve hemocompatibility of the samples. An appealing explanation is that the majority of heparin (which is presented on the polymer before incubating with fresh blood; see Table 1) is removed upon incubation. Namely, bonded heparin always prevents any adhesion and activation of blood platelets on the surface of PET polymer [8]. It is interesting that the concentration of blood platelets on the surface of samples treated by $\mathrm{O}$-atoms and subsequently incubated with heparin appears to be even larger than on the samples treated only by oxygen atoms. A possible explanation of this observation may be deactivation of surface functional groups on the oxygen-treated polymer during incubation with heparin.

Haemostatic response of the PET samples treated by nitrogen atoms (\#4) is similar to that of samples treated by oxygen atoms (\#2). Figure 6 represents images of a sample treated by nitrogen atoms and then incubated with fresh blood, whereas Figure 7 represents those which were also coated with heparin (\#5). The lateral dimension of the spots on the polymer samples is now larger than for samples treated in oxygen plasma afterglow. High magnification images of Figures 6 and 7 also indicate almost complete activation of the adhered blood platelets. Comparison of high-resolution images of Figures 5 and 7 reveals much higher degree of platelet activation for the case of $\mathrm{N}$-atom pretreatment of the sample. The active spots on the nitrogenfunctionalized polymer therefore stimulate rapid activation of the blood platelets. Taking into account huge fluence of 


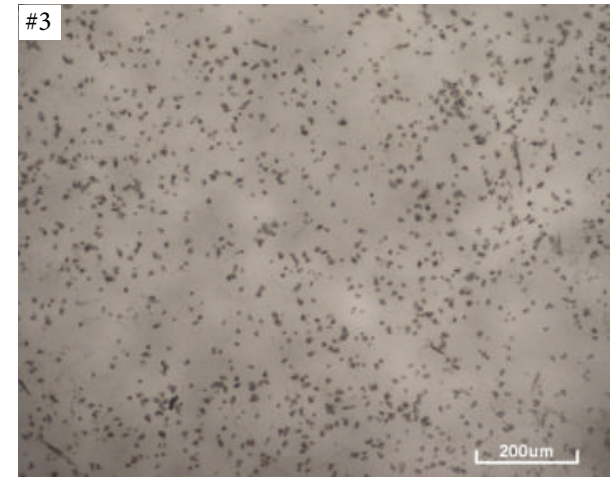

(a)

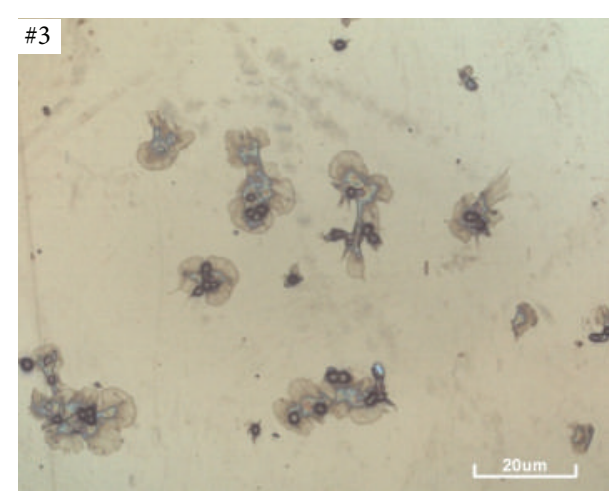

(b)

FIGURE 5: Optical image of platelets on surface of PET polymer treated in oxygen plasma and coated with heparin (\#3) after incubation with fresh blood at two different magnifications ((a) and (b)).

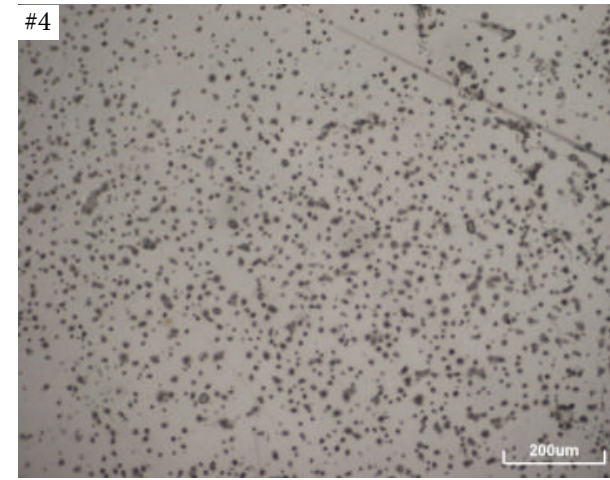

(a)

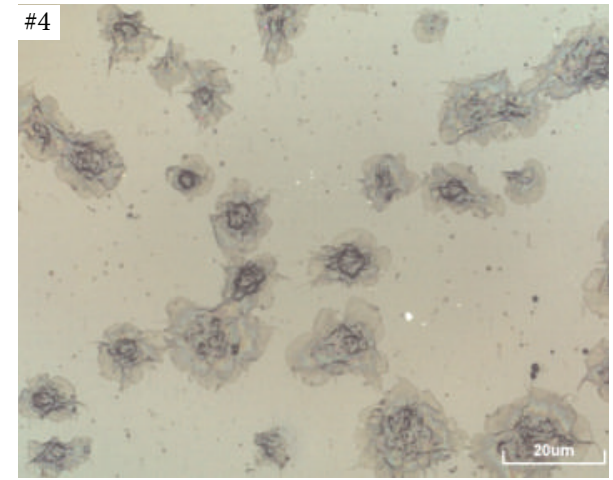

(b)

FIGURE 6: Optical image of platelets on surface of PET polymer treated in nitrogen plasma (\#4) after incubation with fresh blood at two different magnifications ((a) and (b)).

nitrogen atoms it is unlikely to explain rapid activation of blood platelets observed in Figure 6 by lack of nitrogencontaining functional groups. The available analytical techniques, unfortunately, do not allow for high lateral resolution and thus determination of any laterally inhomogeneous distribution of surface functionalities. It is interesting enough that the haemostatic response of the nitrogen-treated sample incubated with heparin (\#5) does not seem to be any better than for the same samples without heparin (\#4). Table 1 doubtfully confirms presence of heparin on such samples. The antithrombogenic coating must be either inhomogeneously distributed onto the sample surface after incubating with fresh blood or removed completely. Taking into account high similarity of images presented in Figures 6 and 7, it is more likely that the heparin was just removed from the polymer surface during incubation with blood. Unfortunately, any surface characterization of polymer after incubation with blood would not give satisfactory information, because blood proteins adhere to the polymer surface and remain bonded well; thus they screen any heparin that may persist at the interface between polymer and protein film.

Finally, let us mention a possible cytotoxic effect of EDC/NHS crosslinker. According to literature, EDC/NHS crosslinker was found to be toxic when added directly to the cell culture medium, because it reacted with the cell DNA [27]. However, cytotoxicity was not observed when EDC/NHS was used as a crosslinker to bind a coating to the surface of implants. Namely, McDade et al. have tested two crosslinkers, EDC and glutaraldehyde, and found glutaraldehyde very toxic, while for EDC cytotoxicity was not observed [28]. Furthermore, also our previous investigation of HUVECs (human umbilical vein endothelial cells) and HMVECs (human microvascular endothelial cells) adhesion on surfaces, where EDC was used as a crosslinker to bind heparin, showed very good cell proliferation [8].

\section{Conclusions}

Samples of PET polymer were pretreated with neutral reactive particles present in the afterglow of nonequilibrium plasma of oxygen and nitrogen, incubated with heparin, and the haemostatic response has been determined by monitoring the density of adhered blood platelets as well as their morphological properties. Flowing afterglow of gaseous plasma has been selected instead of glowing plasma in order to 


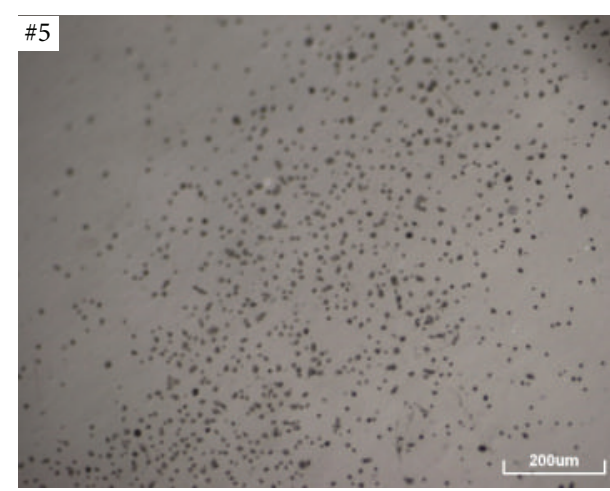

(a)

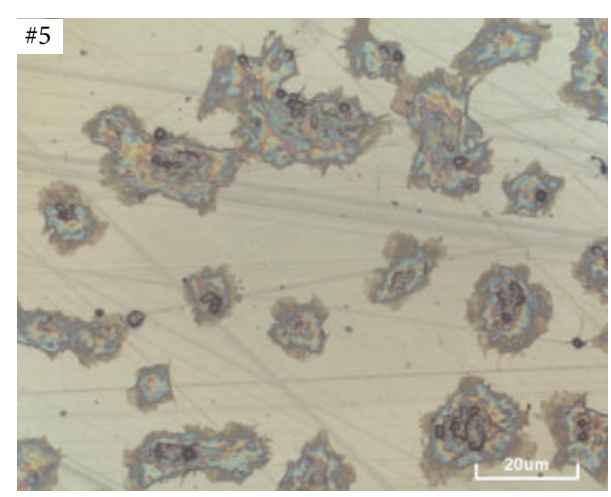

(b)

FIGURE 7: Optical image of platelets on surface of PET polymer treated in nitrogen plasma and coated with heparin (\#5) after incubation with fresh blood at two different magnifications ((a) and (b)).

avoid any modification of polymer surface film by UV/VUV radiation arising from gaseous plasma. The density of neutral reactive particles in the treatment chamber was large enough to assure saturation of any surface functionality that is formed on the polymer surface upon the treatment. The fluence of neutral atoms (either oxygen or nitrogen) was approximately $10^{24} \mathrm{~m}^{-2}$ that is about five orders of magnitude larger than the surface density of atoms in solid materials so even probabilities for interaction as low as $10^{-5}$ should be high enough to saturate surface functionalities. Materials treated in afterglow were tested for haemocompatibility and the results were as good as for materials treated by glowing plasma, if not better. Some differences between samples treated by $\mathrm{O}$ and $\mathrm{N}$-atoms were observed. The density of adhered blood platelets on $\mathrm{O}$-atom treated samples was somehow smaller than on $\mathrm{N}$-atom treated ones. The minimization of blood platelet adhesion was explained by appropriate conformation of blood proteins on polymer surface rich in polar functional groups. The polarity of oxygen groups was even higher than nitrogen; thus the antithrombogenic character of O-treated samples was somehow better than that of Natom treated ones.

Incubation of polymer samples with heparin using the standard technique does not cause substantial effects indicating nonadequate immobilization. The XPS analyses confirmed presence of a thin heparin film on the samples of PET polymer pretreated with $\mathrm{O}$ - or $\mathrm{N}$-atoms, however, not on the untreated polymer samples. The covalent bonding was not achieved in either case, though. The heparin on the surface of samples treated by oxygen or nitrogen plasma afterglow was likely washed away from the polymer surface upon incubation with fresh blood.

Although the treatments described in this paper led to important improvement of polymer biocompatibility when in contact with human blood, the surface finish using this method was not as good as in the case of covalently bonded heparin [8]. The immobilization of such an antithrombogenic polysaccharide coating on the polyethylene surface therefore remains a difficult task.

\section{Conflict of Interests}

The authors declare that there is no conflict of interests regarding the publication of this paper.

\section{Acknowledgment}

This research was financially supported by the Slovenian Research Agency ARRS (Project P2-0082).

\section{References}

[1] J. Jagur-Grodzinski, "Polymers for tissue engineering, medical devices, and regenerative medicine. Concise general review of recent studies," Polymers for Advanced Technologies, vol. 17, no. 6, pp. 395-418, 2006.

[2] T. Holland and A. Mikos, "Review: biodegradable polymeric scaffolds. Improvements in bone tissue engineering through controlled drug delivery," in Tissue Engineering I, K. Lee and D. Kaplan, Eds., vol. 102 of Advances in Biochemical Engineering/Biotechnology, pp. 161-185, Springer, Berlin, Germany, 2006.

[3] S. K. Jaganathan, A. Balaji, M. V. Vellayappan et al., "Review: radiation-induced surface modification of polymers for biomaterial application," Journal of Materials Science, vol. 50, no. 5, pp. 2007-2018, 2015.

[4] A. A. John, A. P. Subramanian, M. V. Vellayappan et al., "Review: physico-chemical modification as a versatile strategy for the biocompatibility enhancement of biomaterials," RSC Advances, vol. 5, no. 49, pp. 39232-39244, 2015.

[5] L. Pinchuk, "A review of the biostability and carcinogenicity of polyurethanes in medicine and the new generation of 'biostable polyurethanes," Journal of Biomaterials Science-Polymer Edition, vol. 6, no. 3, pp. 225-267, 1994.

[6] R. J. Linhardt, S. Murugesan, and J. Xie, "Immobilization of heparin: approaches and applications," Current Topics in Medicinal Chemistry, vol. 8, no. 2, pp. 80-100, 2008.

[7] S. W. Jordan and E. L. Chaikof, "Novel thromboresistant materials," Journal of Vascular Surgery, vol. 45, no. 6, pp. A104-A115, 2007. 
[8] M. Kolar, M. Mozetič, K. Stana-Kleinschek, M. Fröhlich, B. Turk, and A. Vesel, "Covalent binding of heparin to functionalized pet materials for improved haemocompatibility," Materials, vol. 8, no. 4, pp. 1526-1544, 2015.

[9] S. Abou Rich, T. Dufour, P. Leroy, F. Reniers, L. Nittler, and J.-J. Pireaux, "LDPE surface modifications induced by atmospheric plasma torches with linear and showerhead configurations," Plasma Processes and Polymers, vol. 12, no. 8, pp. 771-785, 2015.

[10] M. Gołda, M. Brzychczy-Włoch, M. Faryna, K. Engvall, and A. Kotarba, "Oxygen plasma functionalization of parylene C coating for implants surface: nanotopography and active sites for drug anchoring," Materials Science and Engineering C, vol. 33, no. 7, pp. 4221-4227, 2013.

[11] J. A. Barish and J. M. Goddard, “Topographical and chemical characterization of polymer surfaces modified by physical and chemical processes," Journal of Applied Polymer Science, vol. 120, no. 5, pp. 2863-2871, 2011.

[12] P. Slepicka, N. S. Kasalkova, J. Siegel, Z. Kolska, L. Bacakova, and V. Svorcik, "Nano-structured and functionalized surfaces for cytocompatibility improvement and bactericidal action," Biotechnology Advances, vol. 33, no. 6, pp. 1120-1129, 2015.

[13] S. de Valence, J.-C. Tille, C. Chaabane et al., "Plasma treatment for improving cell biocompatibility of a biodegradable polymer scaffold for vascular graft applications," European Journal of Pharmaceutics and Biopharmaceutics, vol. 85, no. 1, pp. 78-86, 2013.

[14] P. Ferreira, P. Alves, P. Coimbra, and M. H. Gil, "Improving polymeric surfaces for biomedical applications: a review," Journal of Coatings Technology and Research, vol. 12, no. 3, pp. 463475, 2015.

[15] A. Vesel, K. Eleršic, M. Modic, I. Junkar, and M. Mozetic, "Formation of nanocones on highly oriented pyrolytic graphite by oxygen plasma," Materials, vol. 7, no. 3, pp. 2014-2029, 2014.

[16] A. Vesel, I. Junkar, U. Cvelbar, J. Kovac, and M. Mozetic, "Surface modification of polyester by oxygen- and nitrogen-plasma treatment," Surface and Interface Analysis, vol. 40, no. 11, pp. 1444-1453, 2008.

[17] K. Kutasi, V. Guerra, and P. A. Sá, "Active species downstream of an $\mathrm{Ar}-\mathrm{O}_{2}$ surface-wave microwave discharge for biomedicine, surface treatment and nanostructuring," Plasma Sources Science and Technology, vol. 20, no. 3, Article ID 035006, 2011.

[18] M. R. Wertheimer, A. C. Fozza, and A. Holländer, "Industrial processing of polymers by low-pressure plasmas: the role of VUV radiation," Nuclear Instruments and Methods in Physics Research Section B, vol. 151, no. 1-4, pp. 65-75, 1999.

[19] A. Vesel, M. Kolar, N. Recek, K. Kutasi, K. Stana-Kleinschek, and M. Mozetic, "Etching of blood proteins in the early and late flowing afterglow of oxygen plasma," Plasma Processes and Polymers, vol. 11, no. 1, pp. 12-23, 2014.

[20] S. Gore, J. Andersson, R. Biran, C. Underwood, and J. Riesenfeld, "Heparin surfaces: impact of immobilization chemistry on hemocompatibility and protein adsorption," Journal of Biomedical Materials Research. Part B Applied Biomaterials, vol. 102, no. 8, pp. 1817-1824, 2014.

[21] S. Murugesan, J. Xie, and R. J. Linhardt, "Immobilization of heparin: approaches and applications," Current Topics in Medicinal Chemistry, vol. 8, no. 2, pp. 80-100, 2008.

[22] J. Izdebska and T. Sabu, Printing on Polymers: Fundamentals and Applications, Elsevier Science, Waltham, Mass, USA, 2016.

[23] S. L. Goodman, T. G. Grasel, S. L. Cooper, and R. M. Albrecht, "Platelet shape change and cytoskeletal reorganization on polyurethaneureas," Journal of Biomedical Materials Research, vol. 23, no. 1, pp. 105-123, 1989.

[24] M. Modic, I. Junkar, K. Stana-Kleinschek, R. Kostanjšek, and M. Mozetič, "Morphology transformations of platelets on plasma activated surfaces," Plasma Processes and Polymers, vol. 11, no. 6, pp. 596-605, 2014.

[25] M. Modic, I. Junkar, A. Vesel, and M. Mozetic, "Aging of plasma treated surfaces and their effects on platelet adhesion and activation," Surface and Coatings Technology, vol. 213, pp. 98-104, 2012.

[26] N. Recek, M. Jaganjac, M. Kolar et al., "Protein adsorption on various plasma-treated polyethylene terephthalate substrates," Molecules, vol. 18, no. 10, pp. 12441-12463, 2013.

[27] A. B. Moshnikova, V. N. Afanasyev, O. V. Proussakova, S. Chernyshov, V. Gogvadze, and I. P. Beletsky, "Cytotoxic activity of 1-ethyl-3-(3-dimethylaminopropyl)-carbodiimide is underlain by DNA interchain cross-linking," Cellular and Molecular Life Sciences, vol. 63, no. 2, pp. 229-234, 2006.

[28] J. K. McDade, E. P. Brennan-Pierce, M. B. Ariganello, R. S. Labow, and J. Michael Lee, "Interactions of U937 macrophagelike cells with decellularized pericardial matrix materials: influence of crosslinking treatment," Acta Biomaterialia, vol. 9, no. 7, pp. 7191-7199, 2013. 

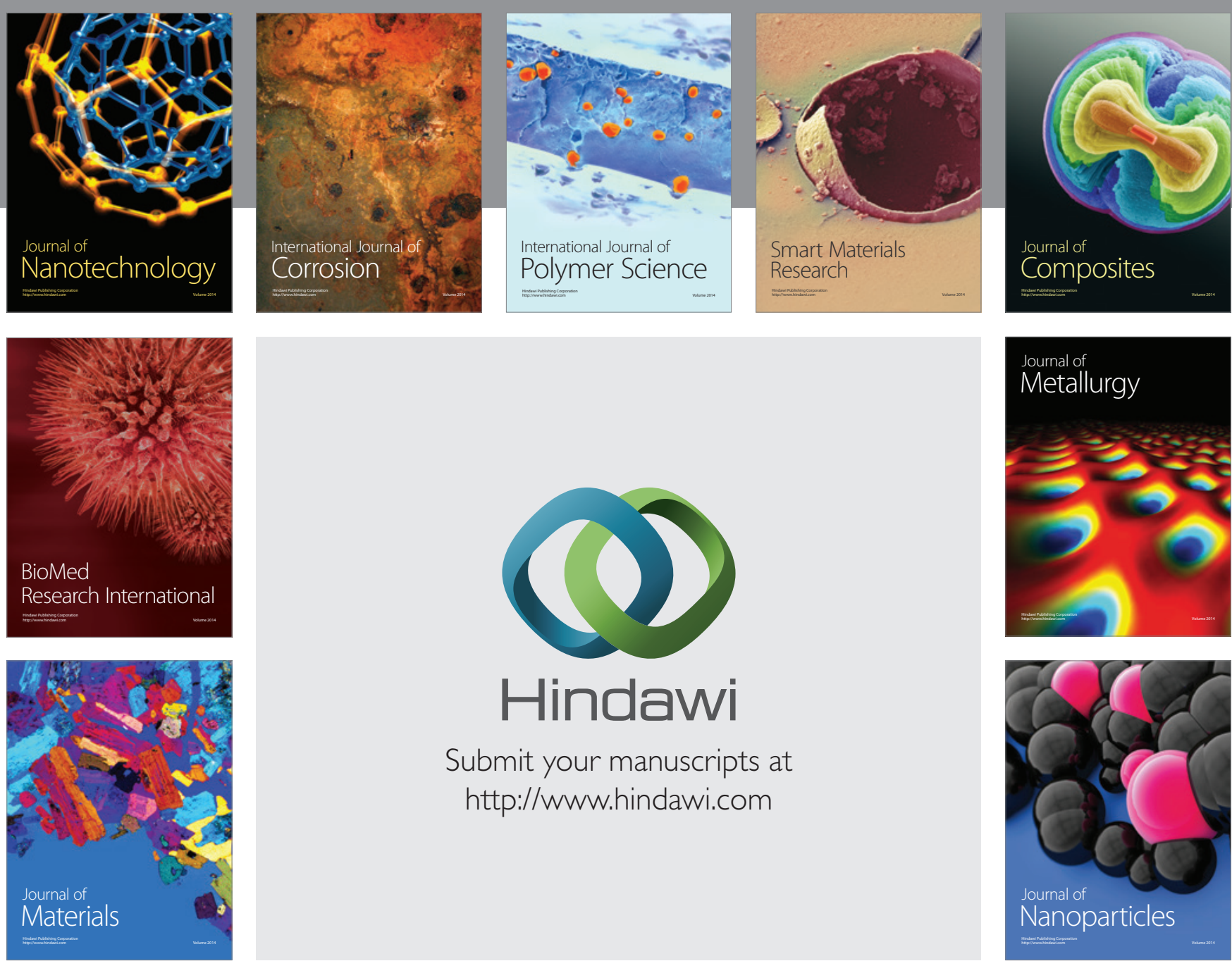

\section{Hindawi}

Submit your manuscripts at

http://www.hindawi.com

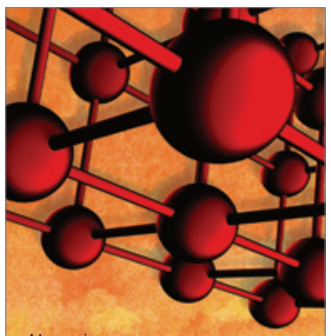

Materials Science and Engineering
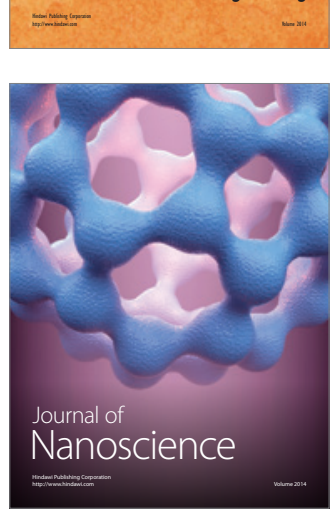
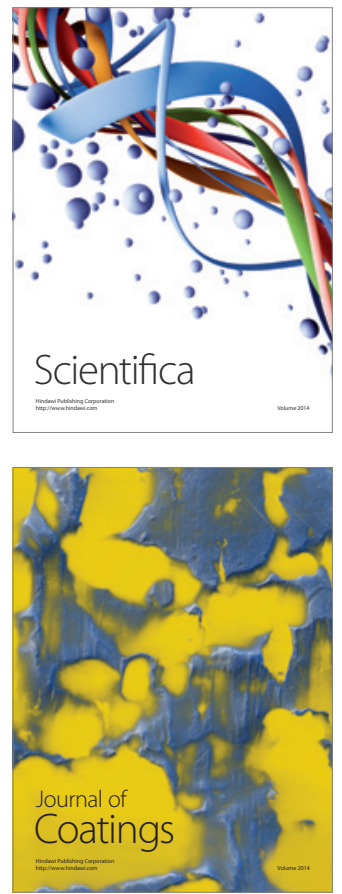
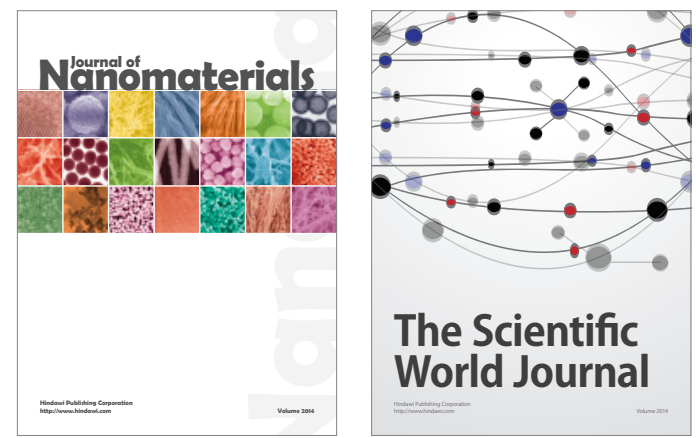

The Scientific World Journal
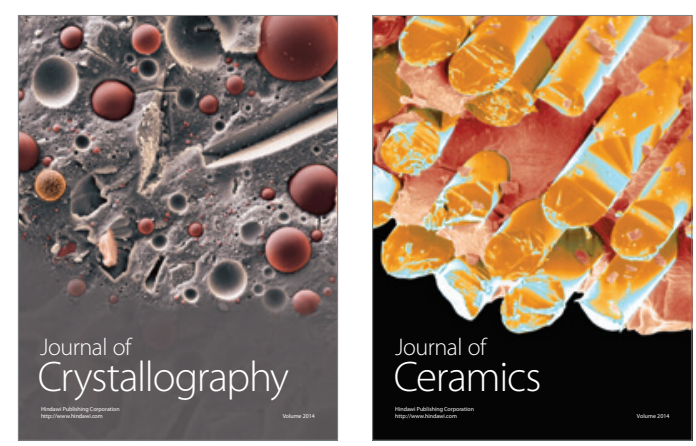
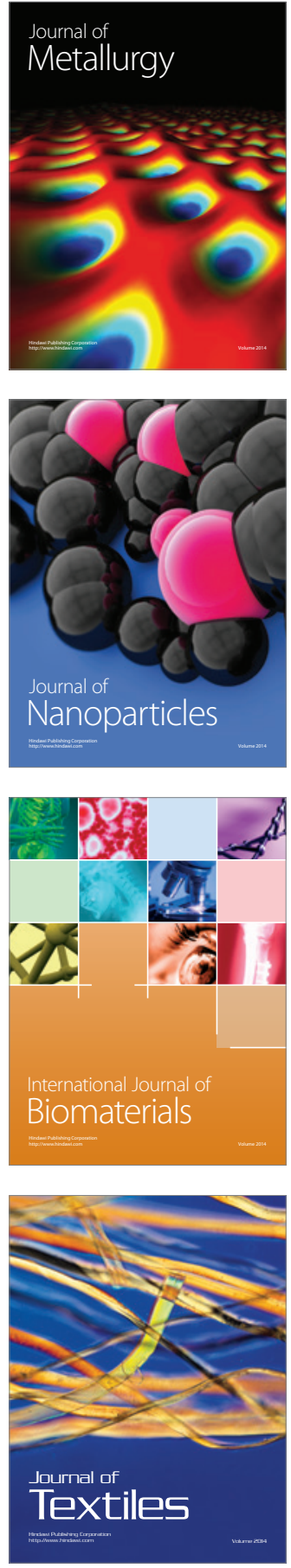